may occur in response to rapid cellular proliferation due to transcellular shift of phosphate from the extracellular fluid into cells whereas longer lasting hypophosphatemia described after treatment with some IV irons seems related to renal phosphate wasting mediated through an increase in the phosphaturic hormone fibroblast growth factor (FGF23). This analysis was done to investigate whether phosphate levels needs to be considered during treatment with iron isomaltoside.

Methods The data presented here is from a pooled analysis of IBD patients from 3 clinical trials of iron isomaltoside performed in IBD patients with IDA. Outcome measures were $s$ phosphate and adverse drug reactions linked to low levels of serum phosphate. Intact FGF23 (iFGF23) was measured in a subgroup of patients.

Results 255 patients (89 men, 166 women) were included in the analysis. Cumulative doses of $\leq 1000 \mathrm{mg}$ or $>1000 \mathrm{mg}$ iron isomaltoside were administered in 189 and 66 patients, respectively.

Hypophosphatemia (s-phosphate $<2 \mathrm{mg} / \mathrm{dL}$ ) was observed in $7.9 \%$ and $6.1 \%$ in patients dosed with $\leq 1000 \mathrm{mg}$ and $>1000 \mathrm{mg}$ iron isomaltoside, respectively $(\mathrm{p}=0.8)$ and $7.4 \%$ for the total population. The drop in phosphate was typically just below $2 \mathrm{mg} / \mathrm{dL}$, appeared one week after the iron infusion and was normalised two weeks after the infusion. These events were asymptomatic, not reported as adverse events, and assessed as non-clinically significant. No severe hypophosphatemia ( $s$-phosphate $<1 \mathrm{mg} / \mathrm{dL}$ ) was observed.

No increase in iFGF23 was seen throughout the study in the subgroup of patients $(n=21)$ where it was measured.

Conclusions No severe hypophosphatemia was observed in IBD patients treated with iron isomaltoside and the small dip in s-phosphate observed among $7.4 \%$ of the patients was independent of dose. Signs of iFGF23-induced renal phosphate wasting were not observed in the sub-population of patients examined.

\section{PWE-050 ANTI-DRUG ANTIBODIES TO ANTI-TNFS: EXPERIENCE AND OUTCOMES AT A TERTIARY IBD CENTRE}

Gregory Sebepos-Rogers*, Nishani Jayasooriya, Radha Gadhok, Hannah Gordon, Sarah O'Donnell. Royal London Hospital, Barts Health NHS Trust, London, UK

\subsection{6/gutjnl-2018-BSGAbstracts.182}

Introduction Therapeutic drug monitoring in the setting of secondary loss of response to anti-TNF therapies has become a routine part of clinical practice in IBD. Decision making following the detection of anti-drug antibodies (ADAs) to Infliximab and Adalimumab remains variable, especially when further therapeutic options are limited and antibody levels are low.

Methods We retrospectively identified all patients with detectable ADAs to Infliximab or Adalimumab between 2014 and 2016 at a tertiary care adult IBD centre, using a drug sensitive assay. Follow-up was to end-2017 evaluating the therapeutic changes made on detection of antibodies, and the clinical outcomes thereafter.

Results 21 of $203(10 \%)$ and 38 of 203 (19\%) tested patients developed ADAs at a median interval of 12 and 8 months after starting therapy with levels above the reported range in
$52 \%(>160 \mathrm{ng} / \mathrm{mL})$ and $50 \%(>200 \mathrm{ng} / \mathrm{mL})$ of Adalimumab and Infliximab patients respectively.

The majority of testing was for suspected loss of response $(46 \%)$ and partial response (15\%). In contrast fewer were for post-induction $(10 \%)$ and yearly reassessment (15\%), of whom $80 \%$ had documented inactive disease. $17 \%$ had experienced an infusion reaction around the time of testing.

For Adalimumab patients (figure 1), 4 had prior ADA to Infliximab. $71 \%$ had a co-prescribed immunomodulator (IM). The mean time from treatment initiation to ADA detection was 23 months. After ADA detection, 8 (38\%) had their Adalimumab therapy continued. Of these, $5(62 \%)$ had dose escalation, of whom 2 had successful ADA suppression and durable remission of 15 and 23 months after initial ADA detection (drug level 1.1 and $4.7 \mathrm{ug} / \mathrm{mL}$ respectively). The remaining 3 had persistent $\mathrm{ADA}$ at next testing. Of the 19 who discontinued Adalimumab, 32\% required a third line out of class biologic.

For Infliximab patients (figure 2), 61\% had a co-prescribed IM. The mean time from treatment initiation to ADA detection was 17 months. After ADA detection, 9 (24\%) continued on Infliximab. Of these, 5 had successful ADA suppression and sustained remission of 14-27 months to the end of the study (drug levels 2.9-9.6 ug/mL). Of the 33 who had a switch in class of biologic, 52\% achieved remission. However, $9 \%$ subsequently required a third line and $6 \%$ a fourth line out of class biologic.

For both groups, neither a high or low level of ADA, nor duration of biologic pre-testing were predictive of outcome.

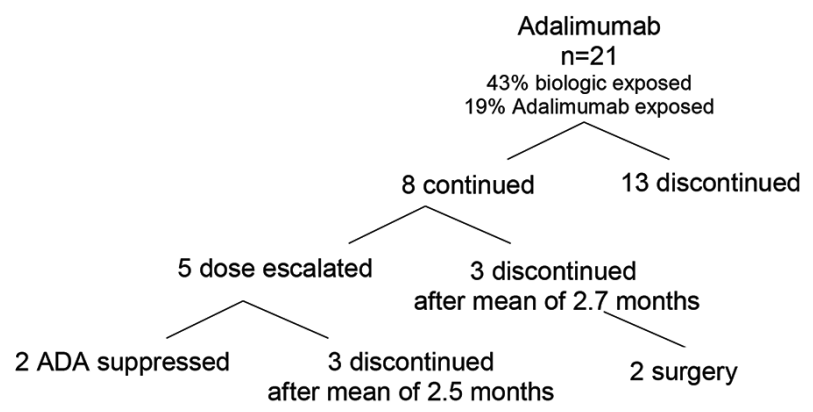

Abstract PWE-050 Figure 1

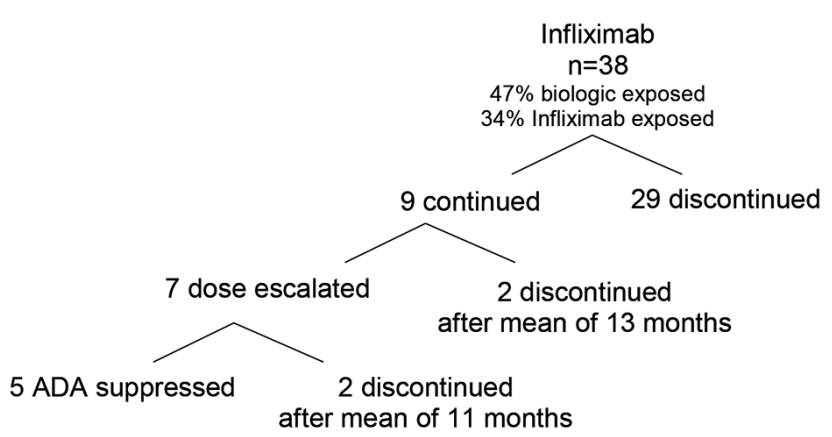

Abstract PWE-050 Figure 2

Conclusions Our study shows some of the challenges in therapeutic drug monitoring when LOR is suspected. When ADAs 
were detected, most patients required a biologic switch in or out of class, and/or surgery, in line with consensus algorithms. However, it appears that in some cases a durable ADA suppression following dose escalation is possible and should be considered when there are limited other therapeutic options.

\section{PWE-051 BIOLOGICAL THERAPY FOR THE TREATMENT OF PRE- POUCH ILEITIS: A RETROSPECTIVE EXPERIENCE FROM THREE CENTRES}

\footnotetext{
${ }^{1,2}$ Jonathan Segal ${ }^{*}{ }^{3}$ Matteo Rottoli, ${ }^{4}$ Richard Felwick, ${ }^{4}$ Simon McLaughlin, ${ }^{3}$ Carlo Vallicelli, ${ }^{1}$ Paul Bassett, ${ }^{1,2}$ Omar Faiz, ${ }^{1,2}$ Ailsa Hart, ${ }^{1,2}$ Susan Clark. 'St Mark's Hospital, Harrow, UK; ${ }^{2}$ Department of Surgery and Cancer, Imperial College, London, UK; ${ }^{3}$ Surgery of the Alimentary Tract, Sant'Orsola - Malpighi Hospital, Alma Mater Studiorum University of Bologna, Bologna, Italy; ${ }^{4}$ The Royal Bournemouth and Christchurch Hospitals, Bournemouth, UK
}

\subsection{6/gutjnl-2018-BSGAbstracts.183}

Introduction Pre-pouch ileitis (PPI) is inflammation of the ileum proximal to an ileoanal pouch, usually associated with pouchitis. This pattern of inflammation can extend for a significant distance proximally. The estimated frequency of PPI is $6 \%$. Symptoms are non-specific but can include increased frequency, obstructive symptoms and bleeding. The treatment of pre-pouch ileitis as a specific entity has been poorly studied, but it is generally treated concurrently with pouchitis. This to our knowledge is the largest study to explore the effectiveness of biologics for the specific treatment of PPI.

Methods This was a retrospective observational study across three centres. Data were collected between January 2008 and February 2018 from two centres in the United Kingdom and one centre in Italy. Patients were censored at the last clinical encounter following their most recent biologic therapy or until they had pouch failure defined by the need for an ileostomy to relieve pouch related symptoms. Patients with PPI treated with a biologic were followed up until last clinical encounter. Outcomes included the presence of PPI following biologic therapy, pouch failure defined by the need for an ileostomy, and remission of PPI defined by the absence of any PPI on endoscopic and histological assessment within a year of biologic therapy.

Result There were 30 patients in our cohort. The median age at diagnosis of IBD was 27 years old (range 6-48). The median time from pouch formation to diagnosis of pre-pouch ileitis was 81.5 months (range 1-147). The median length of time a patient was on biologics at the censorship of the study was 12 months (range 2-62).

On last endoscopic follow-up, 21/30 (70\%) still had endoscopic and histological evidence of PPI, seven had achieved remission and two had no endoscopic follow-up. In our cohort 11 patients had an ileostomy after a median time from starting a biologic of 25 months (range 14-91). Of those who had their UC reclassified to CD, $3 / 10$ (30\%) had pouch failure compared with $8 / 19(42 \%)$ who had UC $(p=0.72)$.

Conclusion Biologics fail to achieve endoscopic and histological remission of PPI in the majority of patients. In a small proportion of patients, they may help to prevent deterioration in symptoms. In a large proportion of patients with pre-pouch ileitis, surgery may be required despite biologic use.
PWE-052 LONG TERM OUTCOMES OF INITIAL IFX THERAPY FOR INFLAMMATORY POUCH PATHOLOGY: A MULTICENTRE RETROSPECTIVE STUDY

1,2Jonathan Segal*, 'Lawrence Penez, ${ }^{1,3}$ Soad Mohsen Elkady, 1,2Guy Worley, ${ }^{4}$ Simon McLaughlin, ${ }^{5}$ Benjamin Mullish, ${ }^{6}$ Mohammed Quraishi, ${ }^{7}$ Nik Ding, ${ }^{7}$ Tamara Glyn, ${ }^{8}$ Kesavan Kandiah, ${ }^{9}$ Mark Samaan, ${ }^{9}$ Peter Inving, ${ }^{1,2}$ Omar Faiz, ${ }^{1,2}$ Susan Clark, ${ }^{1,2}$ Ailsa Hart. ${ }^{1}$ St Mark's Hospital, Harrow, UK; ${ }^{2}$ Department of Surgery and Cancer,Imperial College, London, UK; ${ }^{3}$ Department of Gastroenterology,Faculty of Medicine, Cairo, Egypy; ${ }^{4}$ Department of Gastroenterology, The Royal Bournemouth and Christchurch Hospitals, UK; ${ }^{5}$ Division of Digestive Diseases, St Mary's Hospital Campus, Imperial College, London, UK; ${ }^{6}$ Institute of translational medicine, University of Birmingham, department of gastroenterology, Birmingham, UK; ' Department of Gastroenterology, St Vincent's Hospital, Australia; ${ }^{8}$ Department of Gastroenterology, Queen Alexandra Hospital, Portsmouth, UK; ${ }^{9}$ Department of Gastroenterology, Guy's and St Thomas' NHS Foundation Trust, London, UK

\subsection{6/gutjnl-2018-BSGAbstracts. 184}

Introduction Restorative proctocolectomy is considered the procedure of choice in patients with UC refractory to medical therapy. Inflammation of the pouch is a common complication and in some cases fails to respond to antibiotics, the mainstay of treatment. In such cases, corticosteroid, immunomodulatory or biologic treatments are an option. However, our understanding of the effectiveness of IFX for both chronic pouchitis and Crohn's-like inflammation are based on small studies.

Methods This was an observational, retrospective, multi-centre study to assess the effectiveness IFX for inflammatory disorders related to the pouch. The primary outcome was the development IFX failure defined by primary non-response or secondary loss of response to IFX.

Result 38 patients were included. 20/38 (53\%) who were initiated on IFX for inflammatory disorders of the pouch had IFX failure, 4/38 (11\%) had primary non-response and 16/38 $(42 \%)$ had secondary loss of response with a median followup of 265 days (range 82-2119). Of those that had IFX failure $10 / 20(50 \%)$ avoided an ileostomy by switching to an alternative biologic. In total, 28/38 (74\%) avoided an ileostomy, of these, $17 / 38(45 \%)$ continued on their IFX after a median follow-up of 311 days (42-3968), 5/38 (13\%) were changed to Adalimumab after a median follow-up of 498 days (1-1544), 4/38 (11\%) were changed to Vedolizumab after a median follow-up of 569 (251-1138), 1 achieved histological remission and stopped all treatments at 251 days and 1 was maintained on methotrexate and multiple antibiotics after 3968 days.

Conclusions After initial IFX therapy over half will fail first line IFX, of those that fail half can avoid an ileostomy by switching to an alternative biologic. Patients should be counselled about a high incidence of failure and alternatives should be considered.

\section{PWE-053 BIOFEEDBACK IN PATIENTS WITH ILEOANAL POUCH DYSFUNCTION: A SPECIALIST CENTRE EXPERIENCE}

\footnotetext{
${ }^{1,2}$ Jonathan Segal ${ }^{*}{ }^{1,2}$ Heyson Chan, ${ }^{1}$ Brigitte Collins, ${ }^{1,2}$ Omar Faiz, ${ }^{1,2}$ Professor Ailsa Hart, ${ }^{1,2}$ Susan Clark. 'St Mark's Hospital, Harrow, UK; ${ }^{2}$ Department of Surgery and Cancer, Imperial College, London, UK
}

10.1136/gutjnl-2018-BSGAbstracts. 185

Introduction Restorative proctocolectomy is performed in patients with ulcerative colitis refractory to medical therapy, 